\title{
The solar flare of the 14th of July 2000 (L3+C detector results)
}

\author{
The L3 collaboration ${ }^{\star}$
}

CERN, 1211 Geneva, Switzerland

Received 22 March 2006 / Accepted 8 May 2006

ABSTRACT

Aims. Several experiments have reported observations on possible correlations between the flux of high energy muons and intense solar flares. If confirmed, these observations would have significant implications for acceleration processes in the heliosphere able to accelerate protons and other ions to energies of at least tens of $\mathrm{GeV}$.

Methods. The solar flare of the 14 of July 2000 offered a unique opportunity for the L3+C experiment to search for a correlated enhancement in the flux of muons using the L3 precision muon spectrometer. Its capabilities for observing a directional excess in the flux of muons above $15 \mathrm{GeV}$ (corresponding to primary proton energies above $40 \mathrm{GeV}$ ) are presented along with observations made on the 14th of July 2000.

Results. We report an excess which appeared at a time coincident with the peak increase of solar protons observed at lower energies. The probability that the excess is a background fluctuation is estimated to be $1 \%$. No similar excess of the muon flux was observed up to $1.5 \mathrm{~h}$ after the solar flare time.

Key words. Sun: flares - Sun: coronal mass ejections (CMEs)

\section{Introduction}

It is known that solar protons are accelerated in high energy solar processes, such as solar flares (SFs) and/or coronal mass ejections (CMEs). Solar protons with energies higher than several hundred $\mathrm{MeV}$ can produce secondary particles observed at ground level. These highest energy solar proton events are known as ground level enhancements (GLEs). Since the first observation of a GLE in 1946 (Forbush 1946) more than sixty GLEs have been detected (Shea \& Smart 2001; WebD 2000; Cliver 2006) mainly using the worldwide network of neutron monitors (NMs). More than $100 \mathrm{NMs}$ distributed at different geomagnetic latitudes act as a "geomagnetic spectrometer" for measuring GLEs. Soon after the first observations it was recognized that the Sun can accelerate protons up to $\mathrm{GeV}$ energies (Parker 1957).

It is of interest to know to how high an energy solar protons can be accelerated by the Sun. NMs located at geomagnetic rigidities higher than $10 \mathrm{GV}$ very rarely recorded excesses in GLEs. This shows that for most GLEs the upper energy limit of solar protons indirectly observed by NMs are less than $10 \mathrm{GeV}$. In a very big GLE, on 29th September 1989, the biggest GLE since 1956, the Huancayo NM with a rigidity cut-off of $13 \mathrm{GV}$, observed a significant (>10\%) increase (WebC 1989), suggesting that solar protons with energies higher than $13 \mathrm{GeV}$ are produced.

In recent years, utilizing the technique of particle trajectory tracing (Cramp 1997) with an advanced model of the magnetospheric magnetic field (Tsyganenko 1989), data from different NMs for a same GLE can be well modelled, and the solar proton "beam" approaching the Earth can be well described (Danilova 1999; Duldig 1999; Chebakova 1999). Many such studies showed that solar protons in GLEs often follow a steep spectrum (the typical index of the power spectrum is -6 , or even steeper) and the beam is often anisotropic, sometimes highly anisotropic.

It may thus be expected that the directionally integrating NM may not be sensitive enough to higher energy solar protons with steep spectra and/or anisotropic flux distributions. Therefore, directional detectors (such as muon telescopes) should be better suited for detecting higher energy solar protons in GLEs. Using the underground muon detector Embudo with an equivalent rigidity of $19 \mathrm{GV}$, a positive observation was reported (Swinson 1990) in the GLE of the 29th of September 1989. The signal increase was coincident in time with the increases seen by NMs. It was concluded that the upper rigidity of solar particles present during this event would be approximately $25 \mathrm{GV}$. In the recent review paper of Ryan et al. (2000) it was stated that the solar proton flux in this GLE had to extend up to $20 \mathrm{GeV}$ to produce a positive signal in the Embudo detector, but could not have extended significantly above $30 \mathrm{GeV}$.

However, some exceptional results, mainly obtained from underground muon detectors (Filippov 1991; Alexeyev 1992; Alexeyev 1994; Karpov 1998) have been reported about some possible signals of higher energy solar protons. Among them, the most outstanding one is from the Baksan underground muon detector. This collaboration claimed that the observed excess of the muon flux in the GLE of the 29th of September 1989 was induced by solar protons with energies higher than $500 \mathrm{GeV}$. In spite of its high energy this excess appeared at a time about two hours after the impulsive phase of the flare. In addition, the excess was assumed to be described by a very flat spectrum, because no signal was observed at the same time by the surface detectors with much lower energy threshold.

Thus another question is raised: whether a delayed component of solar protons can be present in a solar flare event. Some analyses of NM data suggest such a picture: besides the acceleration of solar protons during the initial impulsive phase there

* Authorlist at the end, after the references. 
is sometimes a delayed component in some solar flares that can be seen from the time profiles of some NM data (Ryan 2000). It is also of interest to study whether some higher energy muons, like those seen in the Baksan event, appear in the delayed component of a solar flare.

The $\mathrm{L} 3+\mathrm{C}$ collaboration used the precision muon chambers of the L3 spectrometer (Adeva 1990) to measure cosmic ray muons (Adriani 2002; Achard 2004). It possesses many advantages: high directional resolution, high momentum resolution, low momentum threshold and a large sensitive volume. Its running periods (1999-2000) were just during the peak years of the recent solar activity cycle (the cycle No. 23). These factors place this detector in a very good position to observe high energy solar protons and to address the questions discussed above.

One GLE happened during the operation period of $\mathrm{L} 3+\mathrm{C}$, on the 14th of July 2000. In this paper the data analysis and results related to this event are reported. We briefly introduce this event in the next section and the experiment in Sect. 3. The data analysis and results are given in Sect. 4. After discussion of the results in Sect. 5, the conclusion is given in Sect. 6 .

\section{The GLE of the 14th of July 2000}

The GLE of the 14th of July 2000 (Bieber 2002) was associated with an X5.7/3B class solar flare produced in the 9077 sunspot region. The X-ray flare started at 10:03 UT and reached its peak at 10:24 UT. The onset of a type II radio burst, designating the start time of high energy phenomena in the flare, and being thought to be close to the time of relativistic proton acceleration (Cliver 1982, 2006), was at 10:20 UT. Soon after that, at 10:30 UT, the satellite borne detector GOES-8 observed a rapid increase in proton fluxes with energies greater than $10 \mathrm{MeV}$, $50 \mathrm{MeV}$ and $100 \mathrm{MeV}$, respectively (WebB 2000). Observed by the instrument on board the Solar and Heliospheric Observatory $\mathrm{SOHO} / \mathrm{LASCO}$ a full halo, earth-directed CME, was seen to develop during this event (WebA 2000). On ground, more than 20 NMs observed cosmic ray intensity increases ranging from $2 \%$ to $60 \%$ (Usoskin 2000; Flückiger 2001; Belov 2001). The earliest onset time of NMs' increases was at 10:30 UT. Among them, the one having highest geomagnetic rigidity $(4 \mathrm{GV})$ is the Nomnisky NM. A small effect was recorded by the NM at the mountain Alma-Ata station (rigidity $6.7 \mathrm{GV}$ ), indicating that a solar proton flux with energy at least $6.7 \mathrm{GeV}$ has been produced.

This flare was the biggest in the current solar cycle until April 2001, and the third largest solar proton event above $10 \mathrm{MeV}$ since 1976 (Belov 2001). Several studies (Duldig 2001; Pchelkin 2001; Vashenyuk 2001) using the established technique (Cramp 1997) and the standard magnetospheric magnetic field model (Tsyganenko 1989) were made by unifying more than 20 NMs' data in the analysis. This is the first time a GLE was also modelled dynamically as mentioned in (Duldig 2001). In the conclusions the following features were found concerning the solar protons approaching the Earth: the spectrum was soft with a power law index of -6 during the rising phase, of -7 by 11:00 UT, of -8 by 12:00 UT and of -8 to of -9 till 20:00 UT; the pitch angle distribution was derived and it was noted that the particle arrival was anisotropic at the onset time. It then became increasingly isotropic, and after one hour it turned out to be highly anisotropic again. The arrival direction (defined as the pitch symmetry axis) was also rapidly changing with time. All these features guided our analysis described below.

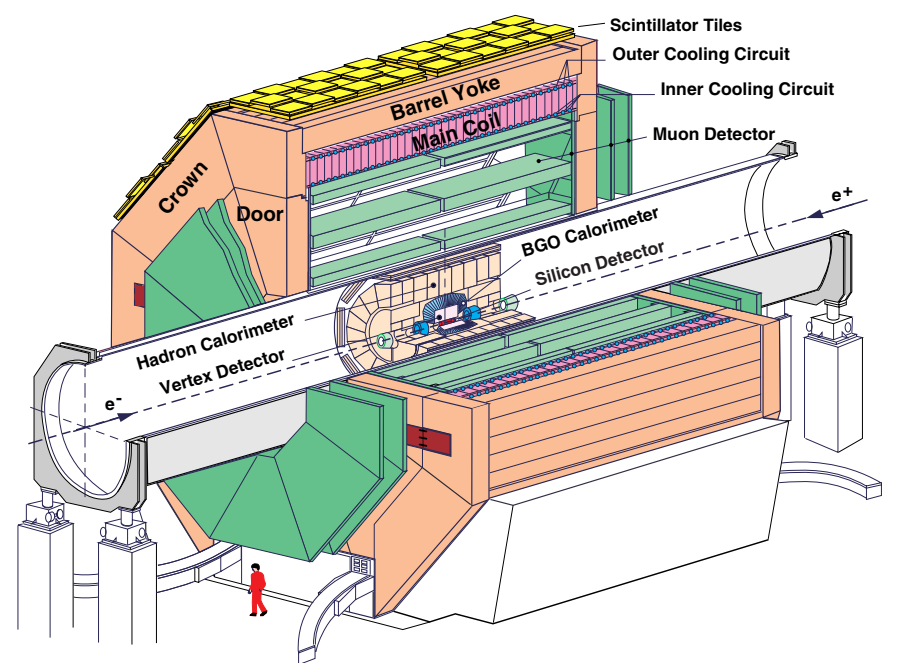

Fig. 1. The L3 spectrometer. Only the muon detectors, the magnet and the scintillator tiles were used in this experiment.

\section{The $L 3+C$ experiment}

The L3+C detector system (Adriani 2002) combined the high precision muon drift chambers of the L3 spectrometer with an air shower array on the surface. A drawing of the spectrometer is shown in Fig. 1. Only the muon detectors, the magnet and the scintillator tiles were used in this experiment. The L3 detector was located at the "Large Electron Positron Collider" LEP, CERN, near Geneva $\left(6.02^{\circ} \mathrm{E}, 46.25^{\circ} \mathrm{N}\right)$ at an altitude of $450 \mathrm{~m}$ above sea level. The vertical geomagnetic rigidity cutoff of the experimental site is $\sim 5 \mathrm{GV}$.

The muon drift-chamber system, with an octant shape in the plane perpendicular to the electron positron beams $(11 \mathrm{~m}$ in width and $11 \mathrm{~m}$ in height) and a square shape in the plane along the beam $\left(11 \mathrm{~m}\right.$ in length), installed in a $1000 \mathrm{~m}^{3}$ magnetic field of $0.5 \mathrm{~T}$, was used to record cosmic ray muons and to measure their momenta. The maximum geometrical acceptance was $\sim 200 \mathrm{~m}^{2} \mathrm{sr}$, covering a zenith angle ranging from $0^{\circ}$ to $\sim 60^{\circ}$. Located underneath approximately $30 \mathrm{~m}$ of molasse the incident muon cutoff energy was $15 \mathrm{GeV}$. This corresponds to primary proton energies above $40 \mathrm{GeV}$. In order to observe cosmic ray events independently of the L 3 experiment, a timing detector composed of $202 \mathrm{~m}^{2}$ of plastic scintillators was installed on top of the magnet, and a separate trigger and DAQ system was used for the data taking.

The dedicated data taking started in 1999 with a trigger rate of about $450 \mathrm{~Hz}$. Up to November 2000 , nearly $1.2 \times 10^{10}$ muon triggers were recorded within an effective live-time of 312 days.

\section{Data analysis and results}

The aim was to search for possible signals of solar protons from our reconstructed muon data set during the period of the solar flare event of the 14th of July 2000, and to see if solar protons exist with higher energies than established until now. As mentioned in Sect. 2 the analysis of NMs' data showed a soft solar proton spectrum in this event, and that the spectrum became softer with time. This means that the higher the solar proton energy the shorter the signal duration. Therefore, this analysis mainly concentrated on a short period, starting from 10:20 UT (the onset time of the type II radio burst), up until the peak time of the increase seen by NMs. The search was first performed to see whether there was an excess muon signal in time 
coincidence with NM data. Then, in order to see whether a delayed component appeared in this event, the data were compared to the background between $\sim 10: 00$ UT and $\sim 12: 00$ UT.

A data set of muons with surface energies between $15 \mathrm{GeV}$ and $25 \mathrm{GeV}$ within the full acceptance of the $\mathrm{L} 3+\mathrm{C}$ detector was used. Setting an upper limit for the surface energies of muons at $25 \mathrm{GeV}$ was motivated by the expected soft solar proton spectrum. An additional search for higher energy signals was also performed.

\subsection{Event selection}

Only events having well reconstructed muon tracks were selected. The following selection criteria were applied:

1. only a single muon track is present in the muon chamber;

2. the track is composed of at least 3 segments of hits in P-chambers (wires parallel to the magnetic field) and by 2 segments of hits in Z-chambers (wires perpendicular to the magnetic field), ensuring good muon track quality.

All selected events were binned according to live-time and to muon's arrival direction on the ground.

A live-time interval of $0.839 \mathrm{~s}$ was used by the $\mathrm{L} 3+\mathrm{C}$ data taking system as a minimal time bin in counting the number of events. 100 such bins were combined to form a 83.9 s live-time bin as the basic time unit in searching for possible signals.

To search for possible directional signals the direction cosines $l=\sin \theta \cos \phi$ and $m=\sin \theta \sin \phi$ were used as measurables of the muon directions, where $\theta$ and $\phi$ are the zenith and azimuth angles of the muon direction at the surface. The squared area of the variables $l$ and $m$ was divided into a $10 \times 10(l, m)$ grid. Ignoring those cells with poor statistics within the detector acceptance, 41 sky cells containing at least 20 events remained for the investigation. The contour lines for directions having an equal event rate are shown in Fig. 2 for data of the 14th of July 2000 .

\subsection{Background}

Data from $12 \mathrm{~h}$ before 10:00 UT were taken as a background measurement. According to the GOES-8 data the event rate of protons with energies larger than $100 \mathrm{MeV}$ was stable during this $12 \mathrm{~h}$ period (WebB 2000), just showing pure background fluctuations. The same event selection criteria, same time binning and direction binning were applied to the background analysis as for the signal search.

\subsection{Result}

The data were compared to the background, while checking possible excesses during the peak time of NMs. In sky cell No.37, defined as $0.2375 \leq l \leq 0.4375,-0.4375 \leq m \leq-0.2375$ (with a solid angle of $0.046 \mathrm{sr}$ ), and for a selected (see below) 16.78 min live-time window (with the real time from 10:24 UT to $10: 42 \mathrm{UT}$ ), an excess of counts in a bin containing 300 events was found (Fig. 3a). This excess was obtained after a first search for an 83.9 s live-time bin (resulting from the on-line live-time counting), starting from 10:20 UT and having an anomalously large number of events followed by other 83.9 s live-time bins which also had a higher number of events. The bin at 10:24 UT that met these requirements was taken as the starting bin for a possible excess. The following 11 live-time bins were combined with it to form the $16.78 \mathrm{~min}$ live-time window (out of 5 trials no other grouping of elementary live-time bins could provide
$\mathrm{N}$

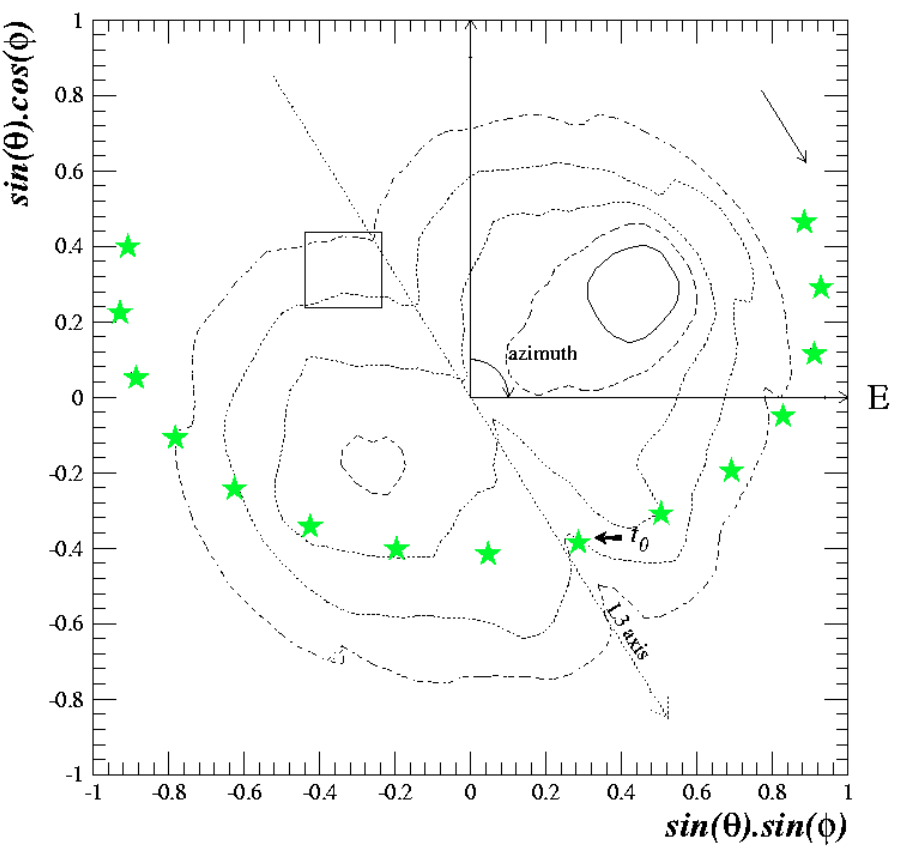

Fig. 2. The distribution of the arrival directions of muons observed by the $\mathrm{L} 3+\mathrm{C}$ detector. The contour lines indicate directions having an equal event rate. The star marks show directions of the Sun for each hour with $t_{0}$ denoting the flare time. The square indicates the sky cell No. 37 (see the text).
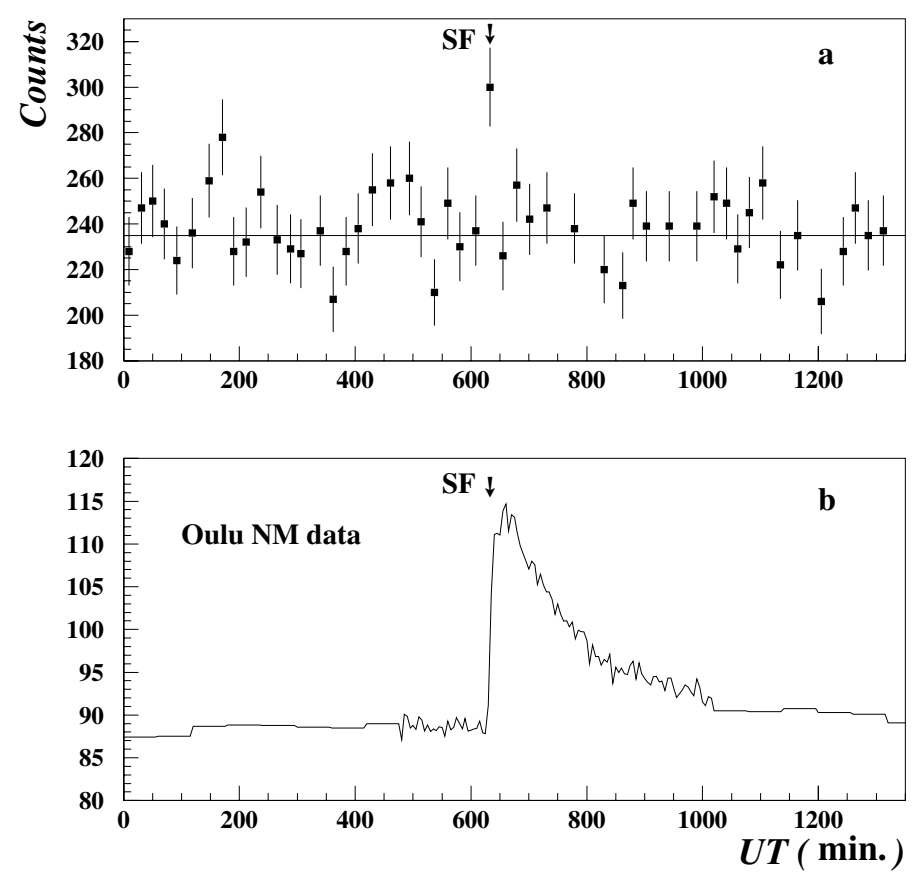

Fig. 3. a) Number of events as a function of time in minutes for the whole day (14th July 2000) in sky cell No. 37. The solar flare time (10:30 UT) is marked by "SF". The live-time bin width is $16.78 \mathrm{~min}$. The solid line shows the mean value of the background. b) Shown is the increase measured by the NM of the Oulu station.

a similar excess of events). The excess appeared at a time just coincident with the peak increase of lower energy solar protons (see the example taken from the the Oulu NM in Fig. 3b). The background distribution in the same sky cell measured 12 hours before 10:00 UT with 16.78 min live-time bins is shown in Fig. 4 


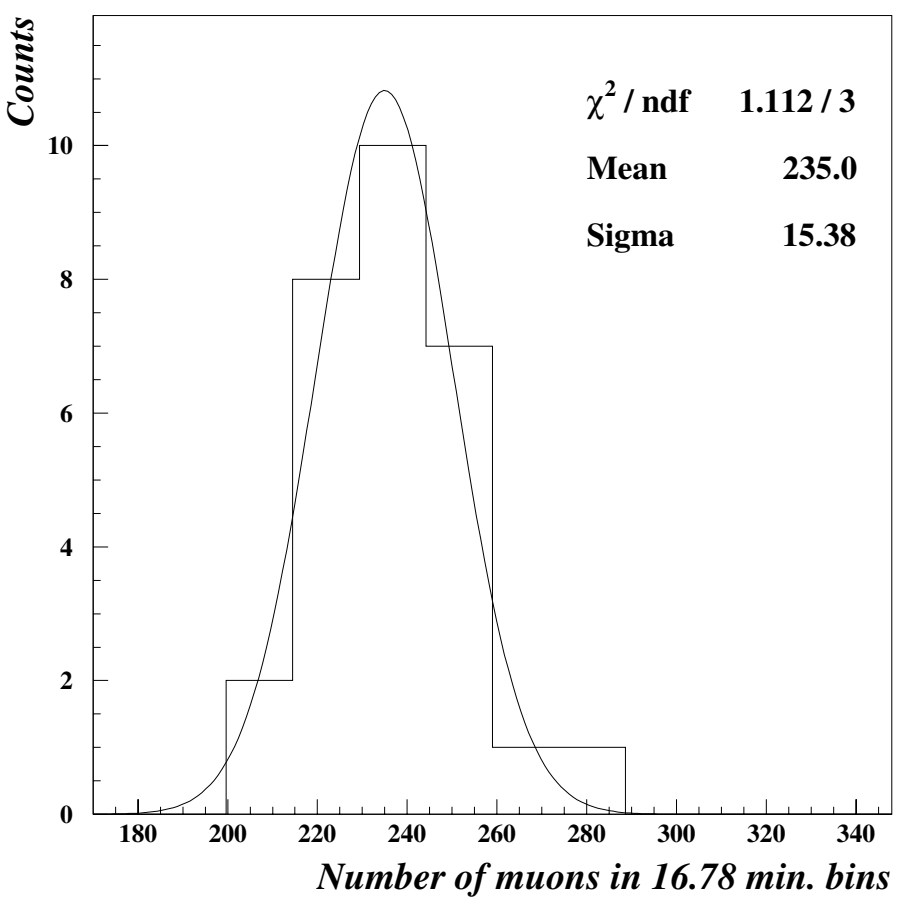

Fig. 4. The distribution of background events in sky cell No. 37 obtained from the 12 hours' data before 10:00 UT.

and is fitted by a Gaussian. Using the fitted mean of 235 and the standard deviation equal to 15.4 , the excess of 65 events gives rise to a $4.2 \sigma$ effect.

It has been also checked whether there were other possible excesses appearing at delayed times. From 9:52 to 11:51 UT five $16.78 \mathrm{~min}$ live-time bins were available corresponding to 9:52-10:23 UT, 10:24-10:42 UT, 10:43-11:06 UT, 11:0711:30 UT and 11:31-11:51 UT, respectively. The histograms of the standard deviations $(\sigma)$ of the counts in all 41 sky cells in these five time intervals are shown in Fig. 5. For each sky cell the $\sigma$ values were calculated from the data and from the fitted background distribution in the same cell. The curve shown in each of the five graphs is a standard Gaussian normalized to the histogram. The deviations between the data and the standard Gaussian can be seen from the $\chi^{2} /$ ndf values that are 3.68/6, $5.73 / 4,5.35 / 6,5.15 / 7$ and 5.52/6, respectively. These values are compatible with the background fluctuation. From Fig. 5 it is also seen that the excess at the peak time of the solar flare and in the sky cell No. 37 is the one having the highest significance.

The lower three figures of Fig. 5 show no excess after the peak increase until 11:51 UT. It means that no evidence of an hourly-delayed signal with short duration, narrow arrival direction and flat energy spectrum is found in this analysis of the 14th July 2000 solar flare.

\section{Discussion}

With the selected live-time binning of $16.78 \mathrm{~min}$, we have found an excess of $4.2 \sigma$ in one of 41 sky cells. The total number of trials being equal to the number of cells times the number of time window selections $(41 \times 5 \times 4=820$; the 5 corresponds to the number of trials to group the elementary live-time bins; the 4 corresponds to the 4 time periods analysed after the solar flare time), the probability for such an excess being due to a background fluctuation is about $1 \%$. An independent analysis of the
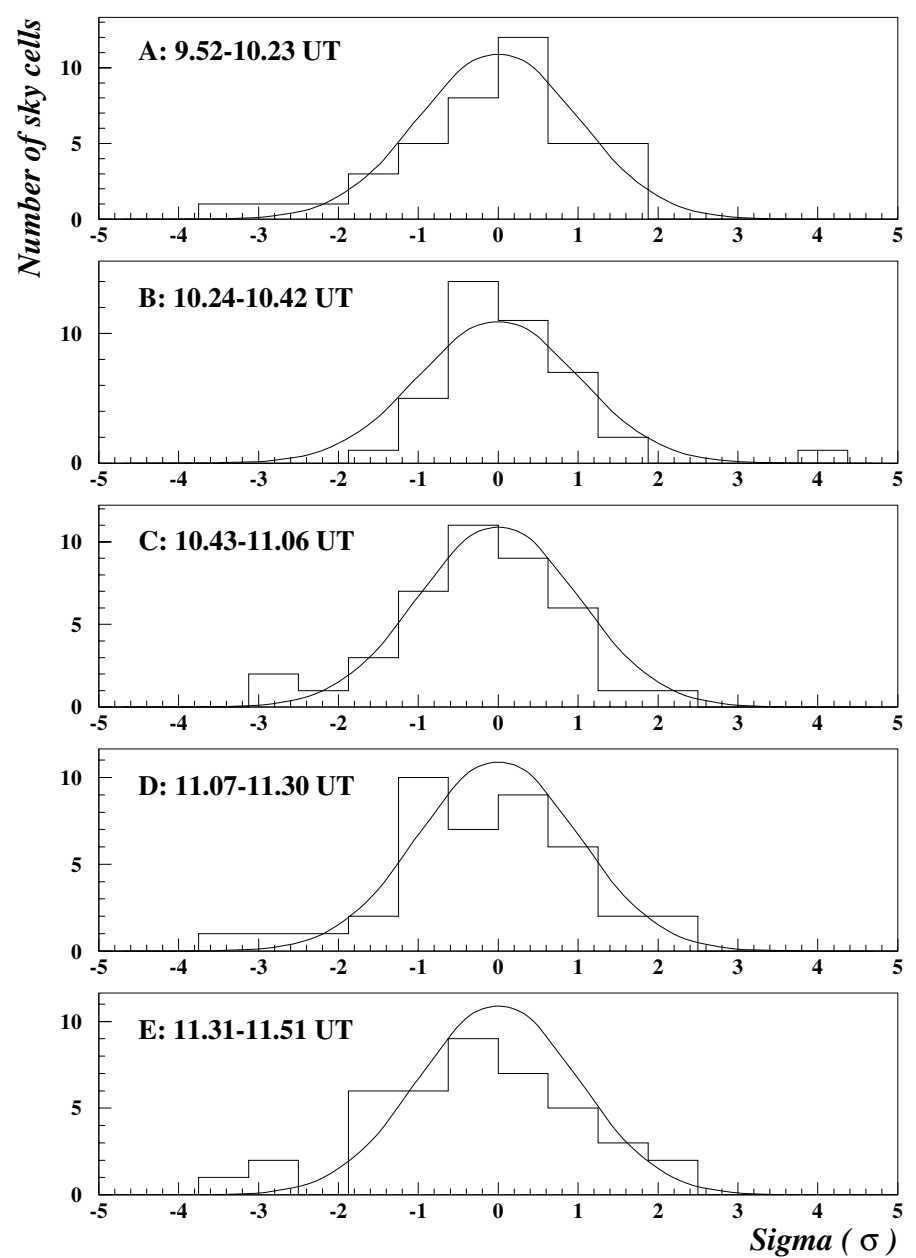

Fig. 5. The shown histograms are distributions of the number of standard deviations from the mean of counts of 41 sky cells, for five time periods from 9:52 UT to 11:51 UT, each with 16.78 min live-time. Curves are standard normal distributions for comparison.

data, using the "running mean" method, provided the same result. A confirmation of this conclusion was obtained by a Monte Carlo generation of 800 distributions of statistical variables and getting the distribution of the maximal standard deviations. One gets also a $1 \%$ probability to find values equal or above 4.2.

By applying the same procedure to muons with energies greater than $25 \mathrm{GeV}$, no excess was seen either in the sky cell No. 37, or at the peak time of increases observed with NMs. This is consistent with a known soft solar proton spectrum deduced from NM data.

In order to estimate the primary energies of solar protons which could be at the origin of the observed excess, a Monte Carlo simulation using the air shower simulation code CORSIKA (Heck 1998) was carried out. Primary protons were assumed to be incident along the directions that make the produced muons to appear in the direction of sky cell No. 37. The index of the primary power law was set to -6 above $20 \mathrm{GeV}$. The energies of the protons which are responsible for the secondary muons in the energy regions $15-25 \mathrm{GeV}$ and arriving at the surface level of $\mathrm{L} 3+\mathrm{C}(450 \mathrm{~m}$ a.s.1.) are distributed in a narrow region: about $85 \%$ of the recorded muons are produced by primary protons ranging from $40 \mathrm{GeV}$ to $100 \mathrm{GeV}$ with a most probable energy of $\sim 70 \mathrm{GeV}$. If the primary spectrum is steeper, this value will be lower. Therefore, this observed "excess" could be attributed to solar protons of energies above those to which a 


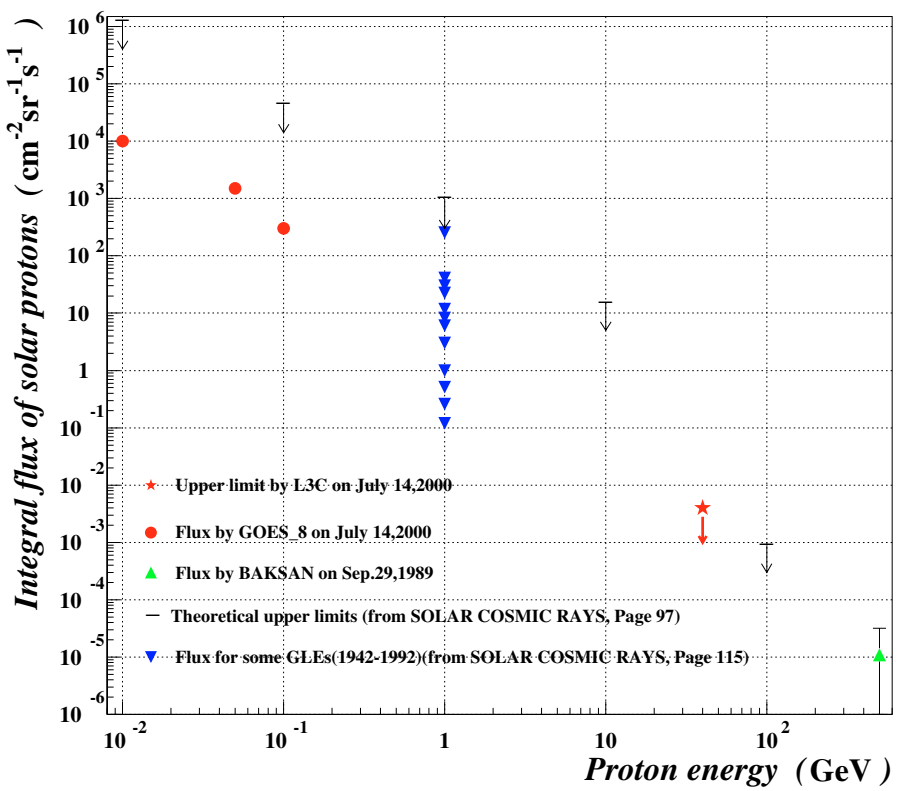

Fig. 6. The upper limit of the solar flare induced proton flux obtained by this work compared with other measurements and theoretical upper limits [29].

set of NMs as a "geomagnetic spectrometer" in the Earth's magnetic field are sensitive to.

An upper limit corresponding to this excess has been estimated. Monte Carlo produced muons reaching the surface were counted, assuming a proton flux with a power spectrum of index -6 and penetrating into the atmosphere from directions around the sky cell No. 37. An area centered around the muon chambers, and large enough to contain the air shower cores, was set, ensuring a very small fraction loss of muons (less than 1\%). Each muon was traced through the molasse and the muon chambers and reconstructed using the same program as for the data. For the background the same simulation procedure was done, but using a primary cosmic ray spectrum with a power law index of -2.7 . As the primary proton flux is known, the comparison between the observed data and the simulated data allows to set an upper limit to the solar proton beam entering the upper atmosphere around the direction of the sky cell No.37:

$I\left(E_{p} \geq 40 \mathrm{GeV}\right) \leq 2.8 \times 10^{-3} \mathrm{~cm}^{-2} \mathrm{~s}^{-1} \mathrm{sr}^{-1}$ (90\% c.1.).

This result is shown in Fig. 6 and is compared to other results (Miroshnichenko 2001).

\section{Conclusions}

In the GLE event of the 14th July 2000 an excess of 65 muons with $E_{\mu}=15-25 \mathrm{GeV}$ over a background of 235 in a particular sky region was observed which lasted from 10:24 UT to 10:42 UT. The chance probability for such an excess to be a background fluctuation is about $1 \%$ in this search. It was timecoincident with the peak increase observed by different NMs during the impulsive phase of the solar flare. If the excess was really induced by solar protons, the observation indicates that solar protons with energies greater than $40 \mathrm{GeV}$ were required to produce the excess.

No evidence of any excess after the peak increase up to 11:51 UT has been observed.
This study shows that a high directional resolution muon spectrometer at a shallow depth may detect solar protons with greater energies than those reported by NMs.

Acknowledgements. We acknowledge the effort of all engineers and technicians who participated in the construction and maintenance of this experiment. I. Usoskin, R. Bütikofer and E. Flückiger are gratefully thanked for providing their NM data and for stimulating discussions. Thanks are also expressed to J. Szabelski for helpful suggestions.

\section{References}

The L3 Collaboration, Achard, P., et al. 2004, Phys. Lett. B, 598, 15

The L3 Collaboration, Adeva, B., et al. 1990, Nucl. Instr. and Meth. in Phys. Res. A, 289, 35

The L3+C Collaboration, Adriani, O., et al. 2002, Nucl. Instr. Meth. Phys. Res. A, 488, 209

Alexeyev, E. N., et al. 1992, Geomagn. Aeron., 32, 192

Alexeyev, E. N., et al. 1994, Geomagn. Aeron., 34, 143

Bieber, J. W., Dröge, W., Evenson, P. A., et al. 2002, ApJ., 567, 622

Belov, A. V., et al. 2001, Proceedings of the 27th ICRC, 2001, 3446

Chebakova, E. A., et al. 1999, Proceedings of the 26th ICRC, 1999, SH 1.7.11

Cliver, E. W. 2006, ApJ, 639, 1206

Cliver, E. W., Kahler, S. W., Shea, M. A., \& Smart, D. F. 1982, ApJ, 260, 362

Cramp, J. L., Duldig, M. L., Flückiger, E. O., et al. 1997, J. Geophys. Res., 102, 24237

Danilova, O. A., et al. 1999, Proceedings of the 26th ICRC, 1999, SH 1.7.08

Duldig, M. L. 2001, Proceedings of the 27th ICRC, 2001, 3363

Duldig, M. L., \& Humble, J. E. 1999, Proceedings of the 26th ICRC, 1999, SH 1.7 .09

Filippov, A. T., et al. 1991, Proceedings of the 22nd ICRC, Dublin, 1991, 3, 113 Flückiger, E. 2001, Private communication, 2001

Forbush, S. E. 1946, Phys. Rev., 70, 771

Heck, D., et al. 1998, Technical report FZKA 6019, 1998, Forschungszentum Karlsruhe

Karpov, S. N., et al. 1998, Nuovo Cim., 21C, 551

Miroshnichenko, L. I. 2001, Solar Cosmic Rays, Kluwer Academic Publishers Parker, E. N. 1957, Phys. Rev., 107, 830

Pchelkin, V. V., et al. 2001, Proceedings of the 27th ICRC, 2001, 3379

Ryan, J. M., Lockwood, J. A., \& Debrunner, H. 2000, Space Sci. Rev., 93, 35

Shea, M. A., \& Smart, D. F. 2001, Proceedings of the 27th ICRC, 2001, 3401

Swinson, D. B., \& Sha, M. A. 1990, Geophys. Res. Lett., 17, 1073

Tsyganenko, N. A. 1989, Planet. Space Sci., 37, 5

Usoskin, I. 2000, Private communication

Vashenyuk, E. V., et al. 2001, Proceedings of the 27th ICRC, 2001, 3383

WebC 1989, http://ulysses.uchicago.edu/NeutronMonitor/Misc/ neutron2.htm

WebB 2000, http://crlhir.crl.go.jp/dimages/gpa1day/proton/ 20000714.gif

WebA 2000, http://crlhir.crl.go.jp/tserdin/plain_report/ 20000714_forecast.html

WebD 2000, http://cosmicrays.oulu.fi/GLE.html

P. Achard ${ }^{21}$, O. Adriani ${ }^{18}$, M. Aguilar-Benitez ${ }^{26}$, M. van den Akker $^{32}$, J. Alcaraz ${ }^{26}$, G. Alemanni ${ }^{24}$, J. Allaby ${ }^{19}$, A. Aloisio ${ }^{30}$, M. G. Alviggi ${ }^{30}$, H. Anderhub ${ }^{52}$, V. P. Andreev ${ }^{6,36}$, F. Anselmo 9 A. Arefiev ${ }^{29}$, T. Azemoon ${ }^{3}$, T. Aziz ${ }^{10}$, P. Bagnaia ${ }^{41}$, A. Bajo ${ }^{26}$, G. Baksay ${ }^{27}$, L. Baksay ${ }^{27}$, J. Bähr ${ }^{51}$, S. V. Baldew ${ }^{2}$, S. Banerjee ${ }^{10}$, Sw. Banerjee ${ }^{4}$, A. Barczyk ${ }^{52,50}$, R. Barillère ${ }^{19}$, P. Bartalini ${ }^{24}$, M. Basile ${ }^{9}$, N. Batalova ${ }^{49}$, R. Battiston ${ }^{35}$, A. Bay $^{24}$, F. Becattini ${ }^{18}$, U. Becker ${ }^{14}$, F. Behner ${ }^{52}$, L. Bellucci ${ }^{18}$, R. Berbeco ${ }^{3}$, J. Berdugo ${ }^{26}$, P. Berges ${ }^{14}$, B. Bertucci ${ }^{35}$, B. L. Betev ${ }^{52}$, M. Biasini ${ }^{35}$, M. Biglietti ${ }^{30}$, A. Biland ${ }^{52}$, J. J. Blaising ${ }^{4}$, S. C. Blyth ${ }^{37}$, G. J. Bobbink ${ }^{2}$, A. Böhm ${ }^{1}$, L. Boldizsar ${ }^{13}$, B. Borgia ${ }^{41}$, S. Bottai ${ }^{18}$, D. Bourilkov ${ }^{52}$, M. Bourquin' ${ }^{21}$, S. Braccini ${ }^{21}$, J. G. Branson ${ }^{43}$, F. Brochu ${ }^{4}$, J. D. Burger ${ }^{14}$, W. J. Burger ${ }^{35}$, X. D. $\mathrm{Cai}^{14}$, M. Capell ${ }^{14}$, G. Cara Romeo ${ }^{9}$, G. Carlino ${ }^{30}$, A. Cartacci ${ }^{18}$, J. Casaus ${ }^{26}$, F. Cavallari ${ }^{41}$, N. Cavallo ${ }^{38}$, C. $\operatorname{Cecchi}^{35}$, M. Cerrada $^{26}$, M. Chamizo ${ }^{21}$, T. Chiarusi ${ }^{18}$, Y. H. Chang ${ }^{47}$, M. Chemarin ${ }^{25}$, A. Chen ${ }^{47}$, G. Chen ${ }^{7}$, 
G. M. $\mathrm{Chen}^{7}$, H. F. $\mathrm{Chen}^{23}$, H. S. Chen ${ }^{7}$, G. Chiefari ${ }^{30}$, L. Cifarelli ${ }^{42}$, F. Cindolo ${ }^{9}$, I. Clare ${ }^{14}$, R. Clare ${ }^{40}$, G. Coignet ${ }^{4}$, N. Colino ${ }^{26}$, S. Costantini ${ }^{41}$, B. de la $\mathrm{Cruz}^{26}$, S. Cucciarelli ${ }^{35}$, R. de Asmundis ${ }^{30}$, P. Déglon ${ }^{21}$, J. Debreczeni ${ }^{13}$, A. Degré ${ }^{4}$, K. Dehmelt ${ }^{27}$, K. Deiters ${ }^{50}$, D. della Volpe ${ }^{30}$, E. Delmeire ${ }^{21}$, P. Denes ${ }^{39}$, F. DeNotaristefani ${ }^{41}$, A. De Salvo ${ }^{52}$, M. Diemoz ${ }^{41}$, M. Dierckxsens ${ }^{2}$, L. K. Ding ${ }^{7}$, C. Dionisi ${ }^{41}$, M. Dittmar ${ }^{52}$, A. Doria ${ }^{30}$, M. T. Dova ${ }^{11, \sharp}$, D. Duchesneau ${ }^{4}$, M. Duda ${ }^{1}$, I. Duran ${ }^{44}$, B. Echenard $^{21}$ A. Eline ${ }^{19}$, A. El Hage ${ }^{1}$, H. El Mamouni ${ }^{25}$, A. Engler ${ }^{37}$, F. J. Eppling ${ }^{14}$, P. Extermann ${ }^{21}$, G. Faber ${ }^{52}$, M. A. Falagan ${ }^{26}$, S. Falciano ${ }^{41}$, A. Favara ${ }^{34}$, J. Fay ${ }^{25}$, O. Fedin ${ }^{36}$, M. Felcini ${ }^{52}$, T. Ferguson ${ }^{37}$, H. Fesefeldt ${ }^{1}$, E. Fiandrini ${ }^{35}$, J. H. Field ${ }^{21}$, F. Filthaut ${ }^{32}$, W. Fisher ${ }^{39}$, G. Forconi ${ }^{14}$, K. Freudenreich ${ }^{52}$, C. Furetta ${ }^{28}$, Yu. Galaktionov ${ }^{29,14}$, S. N. Ganguli ${ }^{10}$, P. Garcia-Abia ${ }^{26}$ M. Gataullin ${ }^{34}$, S. Gentile ${ }^{41}$, S. Giagu ${ }^{41}$, Z. F. Gong ${ }^{23}$, H. J. Grabosch ${ }^{51}$, G. Grenier ${ }^{25}$, O. Grimm ${ }^{52}$, H. Groenstege ${ }^{2}$, M. W. Gruenewald ${ }^{17}$, M. Guida ${ }^{42}$, Y. N. Guo ${ }^{7}$, S. K. Gupta ${ }^{10}$ V. K. Gupta ${ }^{39}$, A. Gurtu ${ }^{10}$, L. J. Gutay ${ }^{49}$, D. Haas ${ }^{5}$, Ch. Haller ${ }^{52}$, D. Hatzifotiadou ${ }^{9}$ Y. Hayashi ${ }^{33}$, Z. X. He ${ }^{8}$, T. Hebbeker ${ }^{1}$, A. Hervé ${ }^{19}$, J. Hirschfelder ${ }^{37}$, H. Hofer ${ }^{52}$, H. Hofer, jun. ${ }^{52}$, M. Hohlmann ${ }^{27}$, A. Holzner ${ }^{52}$, S. R. Hou ${ }^{47}$, A. X. Huo ${ }^{7}$, N. $\operatorname{Ito}^{33}$, B. N. $\operatorname{Jin}^{7}$, P. Jindal ${ }^{15}$, C. L. Jing ${ }^{7}$, L. W. Jones ${ }^{3}$, P. de Jong ${ }^{2}$, I. Josa-Mutuberría ${ }^{26}$, V. Kantserov ${ }^{51, \odot}$, M. Kaur ${ }^{15}$, S. Kawakami ${ }^{33}$, M. N. Kienzle-Focacci ${ }^{21}$, J. K. Kim ${ }^{46}$, J. Kirkby ${ }^{19}$, W. Kittel ${ }^{32}$, A. Klimentov ${ }^{14,29}$, A. C. König ${ }^{32}$, E. $\mathrm{Kok}^{2}$, A. Korn ${ }^{14}$, M. Kopal ${ }^{49}$, V. Koutsenko ${ }^{14,29}$, M. Kräber ${ }^{52}$ H. H. Kuang ${ }^{7}$, R. W. Kraemer ${ }^{37}$, A. Krüger ${ }^{51}$, J. Kuijpers ${ }^{32}$, A. Kunin ${ }^{14}$, P. Ladron de Guevara ${ }^{26}$, I. Laktineh ${ }^{25}$, G. Landi ${ }^{18}$, M. Lebeau ${ }^{19}$, A. Lebedev ${ }^{14}$, P. Lebrun ${ }^{25}$, P. Lecomte ${ }^{52}$, P. Lecoq ${ }^{19}$, P. Le Coultre ${ }^{52, *}$, J. M. Le Goff ${ }^{19}$, Y. Lei ${ }^{3}$, H. Leich ${ }^{51}$, R. Leiste ${ }^{51}$, M. Levtchenko ${ }^{28}$, P. Levtchenko ${ }^{36}$, C. $\mathrm{Li}^{23}, \mathrm{~L} . \mathrm{Li}^{7}$, Z. C. $\mathrm{Li}^{7}$, S. Likhoded ${ }^{51}$, C. H. Lin ${ }^{47}$, W. T. $\operatorname{Lin}^{47}$, F. L. Linde ${ }^{2}$ L. Lista ${ }^{30}$ Z. A. Liu', W. Lohmann ${ }^{51}$, E. Longo ${ }^{41}$, Y. S. Lu Lu C. Luci $^{41}$, L. Luminari ${ }^{41}$, W. Lustermann ${ }^{52}$, W. G. $\mathrm{Ma}^{23}$, X. H. $\mathrm{Ma}^{7}$, Y. Q. $\mathrm{Ma}^{7}$, L. Malgeri ${ }^{19}$, A. Malinin ${ }^{29}$, C. Maña ${ }^{26}$, J. Mans ${ }^{39}$, J. P. Martin ${ }^{25}$, F. Marzano ${ }^{41}$, K. Mazumdar ${ }^{10}$, R. R. McNeil ${ }^{6}$, X. W. Meng ${ }^{7}$, L. Merola ${ }^{30}$, M. Meschini ${ }^{18}$,W. J. Metzger ${ }^{32}$, A. Mihul ${ }^{12}$, A. van Mil $^{32}$, H. Milcent ${ }^{19}$, G. Mirabelli ${ }^{41}$, J. Mnich ${ }^{1}$, G. B. Mohanty ${ }^{10}$, B. Monteleoni ${ }^{18, \dagger}$, G. S. Muanza ${ }^{25}$, A. J. M. Muijs ${ }^{2}$, M. Musy ${ }^{41}$, S. Nagy ${ }^{16}$, R. Nahnhauer ${ }^{51}$, V. A. Naumov ${ }^{18, \infty}$, S. Natale ${ }^{21}$, M. Napolitano ${ }^{30}$, F. Nessi-

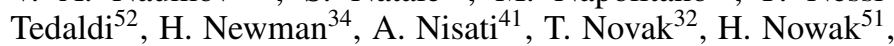
R. Ofierzynski ${ }^{52}$, G. Organtini ${ }^{41}$, I. $\mathrm{Pal}^{49}$, C. Palomares ${ }^{26}$, P. Paolucci ${ }^{30}$, R. Paramatti ${ }^{41}$, J.-F. Parriaud ${ }^{25}$, G. Passaleva ${ }^{18}$, S. Patricelli ${ }^{30}$ T. Paul ${ }^{11}$ M. Pauluzzi ${ }^{35}$, C. Paus ${ }^{14}$, F. Pauss ${ }^{52}$, M. Pedace ${ }^{41}$, S. Pensotti ${ }^{28}$, D. Perret-Gallix ${ }^{4}$, B. Petersen ${ }^{32}$, D. Piccolo ${ }^{30}$, F. Pierella ${ }^{9}$, M. Pieri ${ }^{43}$, M. Pioppi ${ }^{35}$, P. A. Pirouééc, E. Pistolesi ${ }^{28}$, V. Plyaskin ${ }^{29}$, M. Poh ${ }^{21}$, V. Pojidaev ${ }^{18}$, J. Pothier ${ }^{19}$, D. Prokofiev ${ }^{36}$, C. R. Qing ${ }^{8}$, G. Rahal-Callot ${ }^{52}$, M. A. Rahaman ${ }^{10}$, P. Raics ${ }^{16}$, N. Raja ${ }^{10}$, R. Ramelli ${ }^{52}$, P. G. Rancoita ${ }^{28}$, R. Ranieri ${ }^{18}$, A. Raspereza ${ }^{51}$, K. C. Ravindran ${ }^{10}$, P. Razis ${ }^{31}$, S. Rembeczki ${ }^{27}$, D. Ren ${ }^{52}$, M. Rescigno ${ }^{41}$, S. Reucroft ${ }^{11}$, P. Rewiersma $2, \dagger$, S. Riemann ${ }^{51}$, A. Rojkov ${ }^{52,32,18}$, L. Romero ${ }^{26}$, A. Rosca ${ }^{51}$, C. Rosemann ${ }^{1}$, C. Rosenbleck ${ }^{1}$, S. Rosier-Lees ${ }^{4}$, S. Roth ${ }^{1}$, J. A. Rubio ${ }^{19}$, G. Ruggiero ${ }^{18}$, H. Rykaczewski ${ }^{52}$, A. Sakharov ${ }^{52}$, S. Saremi ${ }^{\text {, }}$, S. Sarkar $^{41}$, J. Salicio ${ }^{19}$, E. Sanchez ${ }^{26}$, C. Schäfer ${ }^{19}$ ', V. Schegelsky ${ }^{36}$, B. Schoeneich ${ }^{51}$, D. J. Schotanus ${ }^{32}$, C. Sciacca $^{30}$, L. Servoli ${ }^{35}$, C. Q. Shen ${ }^{7}$, S. Shevchenko ${ }^{34}$, N. Shivarov ${ }^{45}$, V. Shoutko ${ }^{14}$, E. Shumilov ${ }^{29}$, A. Shvorob ${ }^{34}$, D. Son ${ }^{46}$, C. Souga ${ }^{25}$ P. Spillantini ${ }^{18}$, M. Steuer ${ }^{14}$, D. P. Stickland ${ }^{39}$, B. Stoyanov ${ }^{45}$, A. Straessner ${ }^{21}$, K. Sudhakar ${ }^{10}$,
G. Sultanov ${ }^{45}$, L. Z. Sun ${ }^{23}$, S. Sushkov ${ }^{1}$, H. Suter ${ }^{52}$ J. D. Swain ${ }^{11}$, Z. Szillasi ${ }^{27, \pi}$, X. W. Tang ${ }^{7}$, P. Tarjan ${ }^{16}$, L. Tauscher ${ }^{5}$, L. Taylor ${ }^{11}$, B. Tellili ${ }^{25}$, D. Teyssier ${ }^{25}$, C. Timmermans ${ }^{32}$, Samuel C. C. Ting ${ }^{14}$, S. M. Ting ${ }^{14}$, S. C. Tonwar ${ }^{10}$, J. Tóth ${ }^{13}$, G. Trowitzsch ${ }^{51}$, C. Tully ${ }^{39}$, K. L. Tung ${ }^{7}$, J. Ulbricht ${ }^{52}$, M. Unger ${ }^{51}$, E. Valente ${ }^{41}$, H. Verkooijen ${ }^{2}$, R. T. Van de Walle $^{32}$, R. Vasquez ${ }^{49}$, G. Vesztergombi ${ }^{13}$, I. Vetlitsky ${ }^{29}$, G. Viertel ${ }^{52}$, M. Vivargent ${ }^{4}$, S. Vlachos, I. Vodopianov ${ }^{27}$, H. Vogel ${ }^{37}$, H. Vogt $t^{51}$, I. Vorobiev 37,29 , A. A. Vorobyov ${ }^{36}$, M. Wadhwa ${ }^{5}$, R. G. Wang ${ }^{7}$, Q. Wang ${ }^{32}$, X. L. Wang 23 X. W. Wang ${ }^{7}$, Z. M. Wang ${ }^{23}$, M. Weber ${ }^{19}$, R. van Wijk ${ }^{2}$, T. A. M. Wijnen ${ }^{32}$, H. Wilkens ${ }^{32}$, S. Wynhoff ${ }^{39}$, L. Xia ${ }^{34}$, Y. P. Xu ${ }^{52}$, Z. Z. Xu ${ }^{23}$, B. Z. Yang ${ }^{23}$, C. G. Yang ${ }^{7}$, H. J. Yang ${ }^{3}$, M. Yang ${ }^{7}$, X. F. Yang ${ }^{7}$, Z. G. Yao ${ }^{52}$, S. C. $\mathrm{Yeh}^{48}$, Z. Q. $\mathrm{Yu}^{7}$, An. Zalite ${ }^{36}$, Yu. Zalite ${ }^{36}$, C. Zhang ${ }^{7}$, F. Zhang ${ }^{7}$, J. Zhang ${ }^{7}$, S. Zhang ${ }^{7}$, Z. P. Zhang ${ }^{23}$, J. Zhao ${ }^{23}$, S. J. Zhou , G. Y. Zhu ${ }^{7}$, R. Y. Zhu ${ }^{34}$, Q. Q. Zhu', H. L. Zhuang ${ }^{7}$, A. Zichichi ${ }^{9}, 19,20$, B. Zimmermann ${ }^{52}$, M. Zöller ${ }^{1}$, and A. N. M. Zwart ${ }^{2}$

1 III. Physikalisches Institut, RWTH, 52056 Aachen, Germany ${ }^{\S}$

2 NIKHEF, and University of Amsterdam, 1009 DB Amsterdam, The Netherlands

3 University of Michigan, Ann Arbor, MI 48109, USA

4 Laboratoire d'Annecy-le-Vieux de Physique des Particules, LAPP, IN2P3-CNRS, BP 110, 74941 Annecy-le-Vieux Cedex, France

5 Institute of Physics, University of Basel, 4056 Basel, Switzerland

${ }^{6}$ Louisiana State University, Baton Rouge, LA 70803, USA

7 Institute of High Energy Physics, IHEP, 100039 Beijing, PR China ${ }^{\Delta}$

${ }^{8}$ ITP, Academia Sinica, 100039 Beijing, PR China

9 University of Bologna and INFN-Sezione di Bologna, 40126 Bologna, Italy

10 Tata Institute of Fundamental Research, Mumbai (Bombay) 400005 , India

11 Northeastern University, Boston, MA 02115, USA

12 Institute of Atomic Physics and University of Bucharest, 76900 Bucharest, Romania

13 Central Research Institute for Physics of the Hungarian Academy of Sciences, 1525 Budapest 114, Hungary

14 Massachusetts Institute of Technology, Cambridge, MA 02139, USA

15 Panjab University, Chandigarh 160 014, India

16 KLTE-ATOMKI, 4010 Debrecen, Hungary ${ }^{I I}$

17 UCD School of Physics, University College Dublin, Belfield, Dublin 4, Ireland

18 University of Florence and INFN, Sezione di Firenze, 50019 Sesto Fiorentino, Italy

19 European Laboratory for Particle Physics, CERN, 1211 Geneva 23, Switzerland

20 World Laboratory, FBLJA Project, 1211 Geneva 23, Switzerland

21 University of Geneva, 1211 Geneva 4, Switzerland

22 University of Hamburg, 22761 Hamburg, Germany

23 Chinese University of Science and Technology, USTC, Hefei, Anhui 230 029, China $^{\triangle}$

24 University of Lausanne, 1015 Lausanne, Switzerland

25 Institut de Physique Nucléaire de Lyon, IN2P3-CNRS,Université Claude Bernard, 69622 Villeurbanne, France

${ }^{26}$ Centro de Investigaciones Energéticas, Medioambientales y Tecnológicas, CIEMAT, 28040 Madrid, Spainb

27 Florida Institute of Technology, Melbourne, FL 32901, USA

28 INFN - Sezione di Milano, 20133 Milan, Italy

29 Institute of Theoretical and Experimental Physics, ITEP, Moscow, Russia

30 INFN - Sezione di Napoli and University of Naples, 80125 Naples, Italy

31 Department of Physics, University of Cyprus, Nicosia, Cyprus

32 Radboud University and NIKHEF, 6525 ED Nijmegen, The Netherlands 
33 Osaka City University, Osaka 558-8585, Japan

${ }^{34}$ California Institute of Technology, Pasadena, CA 91125, USA

35 INFN - Sezione di Perugia and Università Degli Studi di Perugia, 06100 Perugia, Italy

36 Nuclear Physics Institute, St. Petersburg, Russia

37 Carnegie Mellon University, Pittsburgh, PA 15213, USA

38 INFN-Sezione di Napoli and University of Potenza, 85100 Potenza, Italy

39 Princeton University, Princeton, NJ 08544, USA

40 University of California, Riverside, CA 92521, USA

41 INFN - Sezione di Roma and University of Rome, "La Sapienza", 00185 Rome, Italy

${ }^{42}$ University and INFN, Salerno, 84100 Salerno, Italy

43 University of California, San Diego, CA 92093, USA

${ }^{44}$ University of Santiago de Compostela, 15706 Santiago, Spain

45 Bulgarian Academy of Sciences, Central Lab. of Mechatronics and Instrumentation, 1113 Sofia, Bulgaria

46 The Center for High Energy Physics, Kyungpook National University, 702-701 Taegu, Republic of Korea

47 National Central University, Chung-Li, Taiwan, China

48 Department of Physics, National Tsing Hua University, Taiwan, PR China
49 Purdue University, West Lafayette, IN 47907, USA

50 Paul Scherrer Institut, PSI, 5232 Villigen, Switzerland

51 DESY, 15738 Zeuthen, Germany

52 Eidgenössische Technische Hochschule, ETH Zürich, 8093 Zürich, Switzerland *e-mail: Pierre.Le.Coultre@cern.ch

$\S$ Supported by the German Bundesministerium für Bildung, Wissenschaft, Forschung und Technologie.

* Supported by the Hungarian OTKA fund under contract numbers T019181, F023259 and T037350.

II Also supported by the Hungarian OTKA fund under contract number T026178.

b Supported also by the Comisión Interministerial de Ciencia y Tecnología.

\# Also supported by CONICET and Universidad Nacional de La Plata, CC 67, 1900 La Plata, Argentina.

$\triangle$ Supported by the National Natural Science Foundation of China.

- On leave from the Moscow Physical Engineering Institute (MePhl).

On leave from JINR, 141980 Dubna, Russia.

$\dagger$ Deceased. 